\title{
NÉMATODES PARASITES DES REPTILES DU MEXIQUE
}

\author{
Par Eduardo CABALLERO y $\mathrm{c}$
}

\begin{abstract}
FAMILLE : DIAPHANOCEPHALIDAE Travassos 1919
\end{abstract}
\section{Genre : Kalicephalus Molin 1881}

\section{Kalicephalus humilis n. sp.}

Isolés de l'intestin grêle d'une "nauyaca », Bolhrops atrox, de l'Etat de Veracruz, nous avons étudié deux mâles (dont l'un était incomplet) et sept femelles.

Mâle. - Petit, de $8 \mathrm{~mm}$., 326 de longueur sur $0 \mathrm{~mm}$., 331 à $0 \mathrm{~mm}$., 351 de largeur au niveau de sa portion la plus large, y compris la poche génitale. La cuticule est striée. L'extrémité antérieure est tronquée et oblique, de sorte que la bouche s'ouvre légèrement vers la face dorsale. Cette bouche est pourvue de trois papilles latérales de chaque côté, dont l'une est médiane et deux externes, et d'une capsule dont les parois, dans les régions latérales, mesurent à leur base $61 \mu$ de large. Le diamètre dorso-ventral de l'extrémité céphalique est de $143 \mu$; la capsule buccale mesure $119 \mu$ de large sur $123 \mu$ de longueur. L'œsophage, court et musculeux, est en forme de bouteille et sa partie antérieure mesure $143 \mu$ de longueur sur $102 \mu$ de large et le bulbe $205 \mu$ de longueur sur $151 \mu$ de largeur. L'anneau nerveux est à $225 \mu$ de l'extrémité antérieure et le pore excréteur à $390 \mu$ de la même extrémité.

La poche génitale est symétrique et régulière ; le cône génital est proéminent; la côte dorsale est formée par deux branches terminales à courte bifurcation et par deux sub-terminales qui n'atteignent pas la longueur des terminales; les côtes externes dorsales sont courtes et les côtes qui restent ne présentent aucune particularité. Les spicules sont égaux et mesurent de 546 à $585 \mu$ de longueur sur $16 \mu$ de largeur au niveau de la base ; le gubernaculum a de 139 à $147 \mu$ de longueur sur 12 à $20 \mu$ dans sa partie la plus large.

Femelle. - Plus grande que le mâle, de $15 \mathrm{~mm}$., 950 à $16 \mathrm{~mm}$. de longueur sur 429 à $468 \mu$ de largeur au niveau de sa portion plus

Annales de Parasitologie, T. XVI, $\mathrm{N}^{\circ} 4 .-1^{\text {er }}$ juillet 1938 , p. 327-333. 
large. Le diamètre dorso-ventral de la tête est de $184 \mu$. Les parois chitineuses de la capsule buccale ont une épaisseur de 73 à $78 \mu$ à

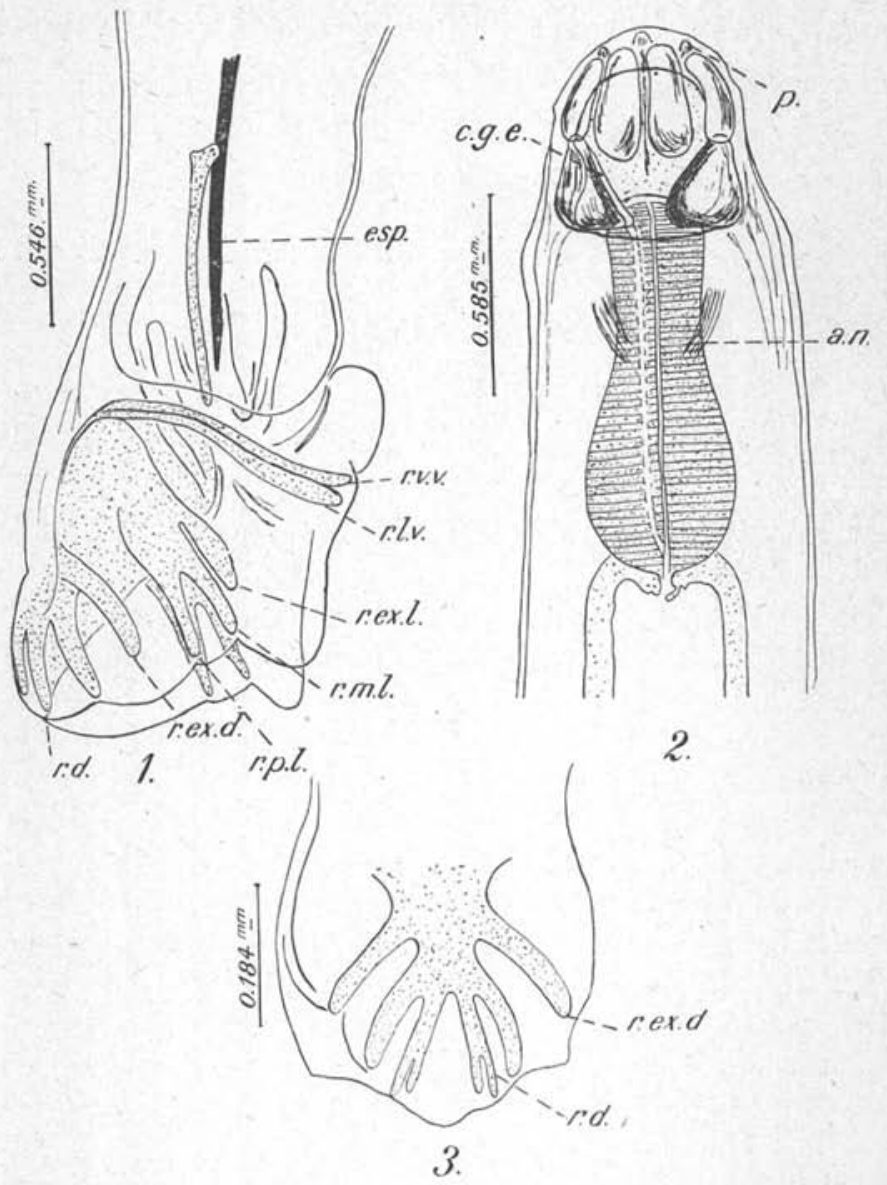

Fig. 1. - Kalicephalus humilis. - 1. Vue latérale de l'extrémité caudale du mâle avec la poche génitale; esp., spicule: r.v.v., côte ventro-ventrale ; r. 1 . v., côte latéro-ventrale ; r. ex. l., côte externo-latérale ; r. m. l., còte moyenne latérale ; r. p. 1 , còte postéro-latérale ; r. ex. d., é̉te externo-dorsale ; r. d., côte dorsale. - 2. Vue latérale de l'extrémité antérieure de la femclle ; p., papille ; c. g. e., conduit de la glande osophagienne ; a. n., anneau nerveux. -3 . Vue ventrale de la poche génitale. Côtes dorsales.

leur base et la capsule elle-même a de 118 à $123 \mu$ de large sur 143 à $164 \mu$ de longueur. La portion antérieure de l'œsophage a de 123 à $131 \mu$ de longueur sur $131 \mu$ de large au commencement et le 
bulbe de 225 à $246 \mu$ de longueur sur 176 à $180 \mu$ de large. L'anneau nerveux est à une distance de 253 à $254 \mu$ de l'extrémité antérieure et le pore excréteur à $351 \mu$ du même point. La partie postérieure s'amincit à mesure qu'on approche de l'extrémité et se termine presque en pointe ; l'anus s'ouvre à $585 \mu$ de cette extrémité.

La vulve a des lèvres peu proéminentes et s'ouvre à la partie postérieure du corps, à $5 \mathrm{~mm}$., 967 de l'extrémité inférieure, et sa rela. tion avec la longueur du corps est de $2,6: 1$.

Les utérus sont divergents ; les œufs, dans l'utérus, renferment un embryon à l'état de morula et mesurent $61 \mu$ de longueur sur $45 \mu$ de largeur.

\section{Hôte. - Bothrops atrox.}

Localisation. - Intestin grêle.

Distribution géographique. - Omealca, Etat de Veracruz, Mexique.

Type. - Collection helminthologique de l'Institut de Biologie de Mexico.

Co-type. - U.S. National Museum. Hel. Coll. No.

Discussion. - L'espèce que nous instituons est très semblable à Kalicephalus subulatus de laquelle elle diffère surtout par la poche génitale ; elle diffère aussi de $K$. agkistrodontis et de $K$. nankingensis par la longueur des spicules et par la relation entre la situation de la vulve et la longueur du corps.

\section{FAMILLE : OXYURIDAE Cobbold 1864}

\section{Genre : Parathelandros Baylis 1930}

\section{Parathelandros scelopori n. sp.}

Les femelles de ce nématode sont abondantes dans l'intestin d'un lézard très commun à Mexico, Sceloporus torquatus; les mâles sont rares. La description a été faite avec un matériel comprenant 15 femelles et 1 mâle.

Mâle. - Très petit, de $2 \mathrm{~mm}$., 106 de longueur, y compris le processus filiforme de l'extrémité postérieure, sur $164 \mu$ de large, sans compter l'aile. La cuticule est striée transversalement. L'extrémité antérieure est étroite et pourvue de trois petites lèvres dans lesquelles se trouvent huit papilles qui entourent la bouche. L'extrémité postérieure est grosse et pourvue d'un processus filiforme non 

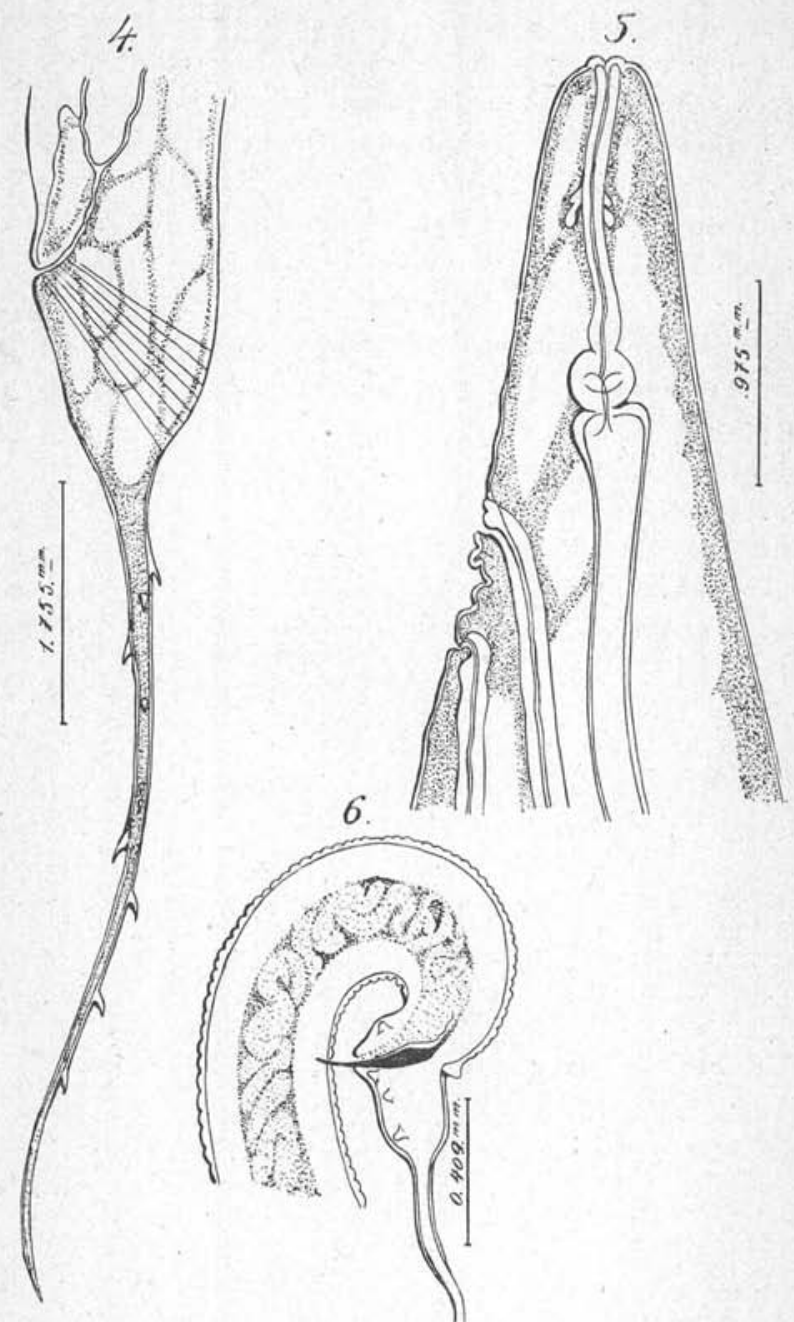

Fig. 2. - Parathelandros scelopori. - 4. Vue latérale de l'extrémité postérieure de la femelle; 5 . Vue latérale de la région antérieure de la femelle ; 6. Vue latérale de la région caudale d'un mâle où on peut voir le spicule et les quatre paires de papilles. 
épineux. Les deux ailes latérales commencent à l'extrémité céphalique et s'étendent jusqu'à l'extrémité postérieure ; elles mesurent $12 \mu$ de large. - L'œesophage commence immédiatement derrière la bouche et a une longueur de $253 \mu$ sur $24 \mu$ de largeur au niveau de sa portion plus élargie. Le bulbe postérieur de l'œsophage est presque sphérique, de $73 \mu, 8$ de longueur sur $697 \mu$ de largeur. L'anneau nerveux est à une distance de $131 \mu$ de l'extrémité antérieure et le pore excréteur à une distance de $653 \mu$ du même point, situé derrière le bulbe œsophagien.

L'extrémité caudale est dépourvue de l'aile correspondante. L'anus s'ouvre à $73 \mu, 8$ de la place où commence le processus filiforme. L'appareil papillaire est formé par une paire de papilles pré-anales et trois paires post-anales, dont deux se trouvent derrière l'anus et l'autre avant la naissance du processus filiforme. Il y a un spicule parfaitement chitinisé, plus large dans sa portion médiane que dans ses extrémités, mesurant $82 \mu$ de longueur sur $8 \mu$ de largeur.

Femelle. - Plus grande que le mâle, elle mesure de 4 mm., 992 à $5 \mathrm{~mm} ., 2$ de longueur sans compter l'appendice filiforme de l'extrémité postérieure, sur $487 \mu$ à $526 \mu$ de largeur dans sa partie la plus large et sans compter l'aile latérale. L'extrémité postérieure est assez large en comparaison avec l'antérieure qui est étroite. Le processus filiforme qu'elle possède mesure de $975 \mu$ à $1 \mathrm{~mm}$., 053 de longueur, portant sur toute sa longueur de 10 à 12 paires d'épines. Le corps est blanc quand elle contient seulement quelques œufs et jaune quand la femelle est complètement gravide. Il paraît être caractéristique des femelles de ce genre d'avoir les ailes latérales très peu développées, puisqu'on fait la même observation sur $P$. mastigurus, $P$. anolis et sur l'espèce ici décrite.

L'osophage mesure de 487 à $507 \mu$ de longueur sur $41 \mu$ de largeur au niveau de sa plus large portion. Le bulbe postérieur de l'œsophage mesure de 127 à $131 \mu$ de longueur sur 139 à $151 \mu$ de largeur. - L'anus est à une distance de 351 à $429 \mu$ du commencement du processus filiforme caudal. L'anneau nerveux se trouve de 135 à $139 \mu$ de l'extrémité antérieure et le pore excréteur à une distance de 780 à $799 \mu$ de la même extrémité.

La vulve est située en avant de la portion médiane du corps, derrière le pore excréteur, et à $897 \mu$ de l'extrémité antérieure ; elle communique avec un court vagin de 65 à $82 \mu$ de large; l'ovojecteur, dirigé en avant, est long et mesure de $1 \mathrm{~mm}$., 053 à $1 \mathrm{~mm} ., 072$. L'utérus est double, et, dans les femelles qui ont atteint leur com- 
plète maturité sexuelle, occupe tout le corps depuis le bulbe de l'œsophage jusqu'à l'extrémité postérieure du corps; dans les femelles non mûres, il va un peu plus loin ; elles sont amphidelphes. Les œufs sont allongés, avec un pôle plus large que l'autre, à capsule double et lisse, de cóuleur jaunâtre, et mesurent de 123 à $135 \mu$ de longueur sur 28 à $33 \mu$ de largeur.

Hôte. - Sceloporus torquatus.

Localisation. - Intestin grêle.

Distribution géographique. - District Fédéral et Misquiahuala, Hgo.

Type. - Collection helminthologique de l'Institut de Biologie de Mexico.

Co-type. - U.S. National Museum Hel. Coll. No.

Discussion. - Il existe actuellement deux espèces de ce genre. Parathelandros scelopori se distingue de P. mastigurus en ce que la femelle possède des épines dans le processus filiforme de l'extrémité caudale et sur les ailes latérales; de $P$. anolis par la présence d'un spicule chez le mâle et par le nombre de papilles.

\section{RÉSUMÉ}

Ce travail constitue la première contribution à la connaissance des nématodes parasites des reptiles du Mexique.- Deux nouvelles espèces sont décrites: Parathelandros scelopori, qui est la troisième espèce de ce genre, et Kalicephalns humilis.

\section{Bibliographie}

BaYLIS (H. A.). - On a collection of Nematodes from Malayan Reptiles. Ann. and Mag. Nat. Hist., Ser. 10, XI, 1933, p. 615.

Boulenger (C. L.). - Report on a collection of parasitic Nematodes, mainly from Egypt. Part. IV. Trichostrongylidae and Strongylidae. Parasitology, XVIII, 1926, p. 86.

Chitwood (B. G.). - Two new Nematodes. Smith. Misc. Coll., XCI, n 11, 1934. Harwood (P. D.). - The Helminths parasitic in the Amphibia and Reptilia of Houston, Texas and vicinity. Proc. U.S. Nat. Mus., LXXXI, art. 17, $\mathrm{n}^{\circ} 2940,1932$.

- Notes on Tennessee helminths. 1. Kalicephalus tennesseenis n. sp. (Nematode). Jour. Tenn. Acad. Sc., IX, 1934, p. 192.

Hsu (H. F.). - On some Kalicephalus species from China with a discussion of certain systematic characters of the genus, Pek, Nat. Hist. Bull., VIII, 1934 , p. 375 . 
Maplestone (P. A.). - Two new species of Nematodes from Indian hosts. Rec. Ind. Mus., XXXI, 1929, part. II; p. 87.

- Parasitic Nematodes obtained from animals dying in the Calcutta Zoological Gardens. Rec. Ind. Mus., XXXIII, 1931, part. II, p. 71.

Ontrepp (R. J.). - Observations on the Nematode genera Kalicephalus, Diaphanocephalus and Occipitodontus g. n., and on the larval devclopment of Kalicephalus philochyadus sp. n. Journ. of Helminthology, I, 1923, p. 165 .

Yamaguti (S.). - Studies on the helminth fauna of Japan. Part. 11. Reptilian Nematodes. Jap. Jour. Zool., VI, 1935, p. 393.

YoRke (W.) et MAplestone (P. A.). - The Nematode parasiles of Verlebrales, 1926.

Laboratoire d'Helminthologie de l'Institut de Biologie de Mexico. 